\title{
A Set of Test Matrices
}

1. Introduction. For the testing of the adequacy and efficiency of any computing method it is clearly very desirable to have readily available a set of reliable test data. The material discussed below has been found by the author to be of great utility for this purpose. It is surmised that it may also be of interest to many others engaged in the testing of new techniques for the inversion of matrices and the determination of characteristic roots and vectors. Furthermore, the results may be of interest also for purely theoretical reasons.

The exact inverses of a sequence of ten nonsymmetric and again of ten symmetric matrices of extremely poor condition, their determinants, and their largest and smallest characteristic roots and associated vectors as well have all been calculated. These are available in the UMT file. These matrices were obtained by a simple modification of the well known Hilbert matrix, and while they share many features characteristic of that matrix, they differ from it in many other important aspects.

The data calculated also permit an examination of the closeness of certain bounds proposed by Ostrowski, PARKER, et al.

2. The sequence of matrices. The set of matrices mentioned above is obtained from the sequence of "positive" matrices.

$$
A_{n} \equiv\left(a_{i j}\right)=\left(\begin{array}{llcc}
1 & 1 & \cdots & 1 \\
2^{-1} & 3^{-1} & & (n+1)^{-1} \\
3^{-1} & 4^{-1} & & (n+2)^{-1} \\
\cdot & \cdot & \cdot \\
\cdot & \cdot & \cdot \\
\cdot & \cdot & & \cdot \\
n^{-1} & (n+1)^{-1} & & (2 n-1)^{-1}
\end{array}\right), \quad n=1,2,3, \cdots
$$

Thus $a_{1 j}=1$ for all $j=1,2,3, \cdots$, and $a_{i j}=(i+j-1)^{-1}$ for $i=2,3, \cdots$; $j=1,2,3, \cdots$.

The matrix $A_{n}$ has the following properties:

a. The determinant $d_{n}$ of $A_{n}$ is of the form $(-1)^{n-1} \delta_{n}^{-1}$, where $\delta_{n}>0$ is an integer.

b. The elements $\alpha_{i j}^{(n)}$ of $A_{n}^{-1}$ are integers. Further,

$$
\sum_{i=1}^{n} \alpha_{i j}^{(n)}= \begin{cases}1 & \text { for } j=1 \\ 0 & \text { for } j \neq 1 .\end{cases}
$$

c. The characteristic root of largest absolute value is positive, that of smallest absolute value negative.

The nonsymmetric matrices $A_{n}$ are thus finite segments of a matrix $A$ which is closely related to the well known symmetric Hilbert matrix

$$
B=\left(b_{i j}\right), \quad b_{i j}=(i+j-1)^{-1}
$$

for $i, j=1,2,3, \cdots$. The matrix $B$ has been discussed in many places, e.g., by Todd [1], and many of its properties are now known. 
In giving brief sketches in substantiation of some of the properties of $A_{n}$ we shall prove first that $\delta_{n}$ satisfies the following recursion formula:

$$
\delta_{n+1}=\left(\begin{array}{c}
2 n \\
n-1
\end{array}\right)\left(\begin{array}{c}
2 n \\
n
\end{array}\right)(2 n+1) \delta_{n}
$$

for $n=1,2,3, \cdots$, with $\delta_{1}=1$.

Multiplying each column of $d_{n+1}$ by the denominator in the last row, and then dividing outside by the product of these factors, yields

$$
d_{n+1}=\frac{1}{(n+1)(n+2) \cdots(2 n+1)}\left|\begin{array}{cccc}
n+1 & n+2 & \cdots & 2 n+1 \\
\frac{n+1}{2} & \frac{n+2}{3} & \cdots & \frac{2 n+1}{n+2} \\
\cdot & \cdot & & \\
\cdot & \cdot & & \\
\cdot & \cdot & & \\
1 & 1 & \cdots & 1
\end{array}\right| .
$$

The following further steps, carried out in the order indicated, lead to (2.2):

1. Subtract the last column from each of the others, making use of the fact that

$$
\frac{n+j}{i+j-1}-\frac{2 n+1}{n+i}=\frac{(n-i+1)(n-j+1)}{(i+j-1)(n+i)}
$$

2. Remove from the first $n$ columns the respective factors $n, n-1, \cdots, 1$ to the outside, and reduce the determinant to the order $n$.

3. Finally remove from all the rows except the first the respective factors

getting

$$
\frac{n-1}{n+2}, \frac{n-2}{n+3}, \cdots, \frac{1}{2 n},
$$

$$
\begin{aligned}
d_{n+1} & =-\frac{n !(n-1) !}{(n+1)(n+2) \cdots(2 n+1)(n+2)(n+3) \cdots 2 n} d_{n} \\
& =-\left[\frac{(n-1) !}{(n+2) \cdots(2 n)}\right]^{2} \cdot \frac{n}{(2 n+1)(n+1)} d_{n} \\
& =-\left(\begin{array}{c}
2 n \\
n-1
\end{array}\right)^{-2} \cdot \frac{n}{(2 n+1)(n+1)} d_{n},
\end{aligned}
$$

which proves (2.2).

The solution of the difference formula (2.3) leads to

$$
d_{n}=(-1)^{n-1} \frac{[1 ! 2 ! \cdots(n-2) !(n-1) !]^{3}}{(n-1) !(n+1) !(n+2) ! \cdots(2 n-1) !}
$$

for $n=1,2,3, \cdots$.

Consequently,

$$
\operatorname{det} A_{n}=(-1)^{n-1} \frac{[1 ! 2 ! \cdots(n-1) !]^{4}}{1 ! 2 ! \cdots(2 n-1) !} n .
$$


In [1] it was shown that for the finite segment $B_{n}$ of order $n$ of Hilbert's matrix,

so that

$$
\operatorname{det} B_{n}=\frac{[1 ! 2 ! \cdots(n-1) !]^{4}}{1 ! 2 ! \cdots(2 n-1) !},
$$

$$
\operatorname{det} A_{n}=(-1)^{n-1} n \operatorname{det} B_{n} .
$$

The value of $d_{n}$ may be estimated quite easily using (2.2) and Stirling's formula $n ! \sim(n / e)^{n}(2 \pi n)^{1 / 2}$; the result is

$$
\left|d_{n}\right| \sim 3^{n} 2^{-2 n(n-1)} \phi(n),
$$

with $\phi(n)$ of the form $C_{0} n^{2}, C_{0}$ denoting a constant.

\section{TABLE 1a. Values of $\delta_{n}$}

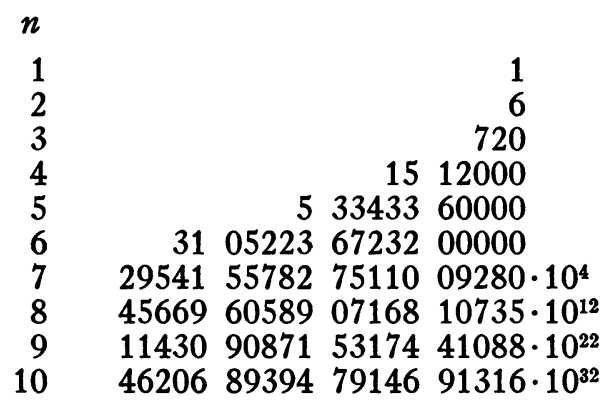

TABLE 1b. Measures of condition

$\begin{array}{rccc}n & d\left(A_{n}\right) & M\left(A_{n}\right) & P\left(A_{n}\right) \\ 1 & 1 & 1 & \\ 2 & 6^{-1} & 12 & 12 \cdot 587 \\ 3 & 720^{-1} & 540 & 354 \cdot 51 \\ 4 & \left(15120 \cdot 10^{2}\right)^{-1} & 17280 & 13090 \\ 5 & \left(53343 \cdot 10^{6}\right)^{-1} & 67200 \cdot 10^{1} & 45057 \cdot 10^{1} \\ 6 & \left(31052 \cdot 10^{12}\right)^{-1} & 23814 \cdot 10^{3} & 15259 \cdot 10^{3} \\ 7 & \left(29542 \cdot 10^{19}\right)^{-1} & 80681 \cdot 10^{4} & 51270 \cdot 10^{4} \\ 8 & \left(45670 \cdot 10^{27}\right)^{-1} & 28333 \cdot 10^{6} & 17164 \cdot 10^{6} \\ 9 & \left(11431 \cdot 10^{37}\right)^{-1} & 95447 \cdot 10^{7} & 57364 \cdot 10^{7} \\ 10 & \left(46207 \cdot 10^{47}\right)^{-1} & 33640 \cdot 10^{9} & 19158 \cdot 10^{9}\end{array}$

A listing of the values $\delta_{n}$ for $n=1,2, \cdots, 10$, rounded to 20 significant figures, is given in Table 1a. The extremely rapid increase of $\delta_{n}$ is worthy of note; it serves to underline the poor "condition" of the $A_{n}$, as measured in terms of $d_{n}$. As will be shown later other, more suitable measures of condition,

$$
\begin{aligned}
& M(A)=n \cdot\|A\|\left\|A^{-1}\right\|, \quad\|A\|=\max _{i, j}\left|a_{i j}\right| \\
& P(A)=\left|\lambda_{\max }(A) / \lambda_{\min }(A)\right|,
\end{aligned}
$$

are indicative of the same phenomenon.

Next a few remarks about the inverses $A_{n}{ }^{-1}$. All the elements of $A_{n}{ }^{-1}$ are 
integers. In fact, it can be shown that for $i=1,2, \cdots$, we have

$$
\alpha_{i 1}^{(n)}=(-1)^{n-i}\left(\begin{array}{c}
n+i-1 \\
i-1
\end{array}\right)\left(\begin{array}{l}
n \\
i
\end{array}\right)
$$

while for $i=2,3, \cdots$ and $j=1,2, \cdots, n$, we have

where

$$
\alpha_{i, j+1}^{(n)}=(-1)^{i-i} k_{i, j+1}\left(\begin{array}{c}
n+i-1 \\
i+j
\end{array}\right)\left(\begin{array}{c}
n+j \\
i+j
\end{array}\right)
$$

$$
k_{i, j+1}=\left(\begin{array}{c}
i+j \\
j
\end{array}\right)\left(\begin{array}{c}
i+j-1 \\
j-1
\end{array}\right) i \text {. }
$$

Let us proceed to the characteristic roots $\lambda_{i}$ and characteristic vectors $x_{i}$ of $A_{n}$, arranging the $\lambda_{i}$ according to increasing absolute value: $\left|\lambda_{1}\right| \leq\left|\lambda_{2}\right| \leq \cdots \leq\left|\lambda_{n}\right|$; we shall set $\lambda_{1} \equiv \lambda_{m}, \lambda_{n} \equiv \lambda_{M}$. Let the vector $x_{i}$ have the components $x_{i j}$, $j=1,2, \cdots, n$.

Since

$$
\operatorname{det}[A-\lambda I]=(-1)^{n} \lambda\left[\lambda^{n-1}-\left(\Sigma_{i} a_{i i}\right) \lambda^{n-2}+\cdots\right]+(-1)^{n-1} \delta_{n}^{-1},
$$

it follows immediately that there is always at least one positive characteristic root. It seems that there is only one positive characteristic root, the others being negative.

The existence of a positive characteristic root also follows from the result of FROBENIUS [2] that the root $\lambda_{M}$ of largest absolute value of any positive nondecomposable matrix is positive and simple, and may be associated with a proper vector having positive components only, the only vector possible that possesses this property.

Estimates for the $\lambda_{i}$ are readily calculated from the following theorem due to PARKer [3]:

Let $U=\left(u_{i j}\right)$ be an arbitrary matrix of order $n$. Put

$$
\xi=n^{-1} \sum_{i=1}^{n}\left|u_{i i}\right| ; \quad P_{i}=\sum_{j=1}^{n}\left|u_{i j}\right|, \quad Q_{j}=\sum_{i=1}^{n}\left|u_{i j}\right| ;
$$

$$
S_{i}=P_{i}+\left|u_{i i}-\xi\right|, \quad S=\max _{i} S_{i} ; \quad T_{j}=Q_{j}+\left|u_{j j}-\xi\right| ; \quad T=\max _{j} T_{j} .
$$

Then for every characteristic root $\lambda$ of $U$

$$
|\lambda-\xi| \leq \min (S, T) \text {. }
$$

Furthermore, the number $\xi$ may be replaced by any other number.

Applying (2.5) to the matrix $A_{n}$, for $\xi=0$, it is seen that the maximum column sum $T$ is a bound:

$$
\lambda_{M}^{(n)}<\log (n+1)+\gamma,
$$

with $\gamma=0.577 \cdots$ denoting Euler's constant.

Bounds for the components of $x_{M}$ may be obtained by means of Ostrowski's 
theorem [4]:

Let $A_{n}=\left(a_{i j}\right)$ be a matrix of order $n$ of positive elements. Put

$$
\begin{aligned}
& R_{i}=\sum_{j=i}^{n} a_{i j}, \quad i=1,2, \cdots, n ; \quad R=\max _{i} R_{i}, \quad r=\min _{i} R_{i}: \\
& k_{1}=\min _{i} a_{i i}, \quad k_{2}=\min _{i \neq j} a_{i j} ; \quad \sigma_{1}=\left[\left(r-k_{1}\right) /\left(R-k_{1}\right)\right]^{1 / 2} .
\end{aligned}
$$

Further, let the positive components $x_{M j}$ of the characteristic vector $x_{M}$ belonging to $\lambda_{M}$ be normalized so that

Then

$$
x_{M n} \leq x_{M, n-1} \leq \cdots \leq x_{M 2} \leq x_{M 1}=1
$$

provided $R \neq r$.

$$
k_{2} /\left(R-r+k_{2}\right)<x_{M n} \leq \sigma_{1}
$$

In our case

$$
R=n, \quad k_{1}=(2 n-1)^{-1}, \quad k_{2}=(2 n-2)^{-1}, \quad r=\sum_{j=1}^{n}(n+j-1)^{-1} .
$$

It is found that

$$
\left(2 n^{2}\right)^{-1}\left[1+\mathrm{O}\left(n^{-1}\right)\right]<x_{M n}<[\log 2 / n]^{1 / 2}\left[1+\mathrm{O}\left(n^{-1}\right)\right] .
$$

The characteristic roots of least absolute value $\lambda_{m}$ are frequently also of interest. If the $\lambda_{i}{ }^{(n)}$ are distinct, then

$$
A_{n}{ }^{-1}=\sum_{i=1}^{n}\left(1 / \lambda_{i}{ }^{(n)}\right) x_{i}{ }^{(n)} \cdot r_{i}{ }^{(n)}
$$

where the $x_{i}{ }^{(n)}$ denote the characteristic column vectors of $A_{n}$ belonging to $\lambda_{i}{ }^{(n)}$, and the $r_{i}^{(n)}$ are the characteristic row vectors of the transposed matrix $A_{n}{ }^{T}$ belonging to $\lambda_{i}{ }^{(n)}$. The smallest root $\lambda_{m}{ }^{(n)}$ may then be found as the dominant root $\mu_{M}^{(n)}$ of $A_{n}^{-1}$.

If it is further known that the roots $\lambda$ of the matrix $U=\left(u_{i j}\right)$ are real, then (2.5) permits the conclusion

$$
\lambda \geq \xi-\min (S, T)
$$

if, in addition, the particular $\operatorname{root} \lambda=\mu_{M}$ is negative, then clearly

$$
\mu_{M}^{-1} \leq[\xi-\min (S, T)]^{-1} .
$$

Another upper bound for $\lambda_{m}$ is provided by the fact that for definite matrices Thus

$$
(n\|A\|)^{-1} \leq\left\|A^{-1}\right\| \leq\left|\lambda_{m}\right|^{-1} \text {. }
$$

$$
\left|\lambda_{m}\right| \leq\left\|A^{-1}\right\|^{-1} \text {. }
$$

3. The inverses and determinants. The first ten inversions were carried out by the method of partitioning [5]. A tabulation of the inverse $A_{6}{ }^{-1}$ computed by this method is given in Table 2 . 
TABLE 2. The inverse of $A_{6}$

$$
A_{b^{-1}}{ }^{-1}=\left(\begin{array}{rrrrrrrrrr}
6 & 630 & - & 6720 & 22680 & -30240 & & 13860 \\
105 & -7350 & 88200 & -3 & 17520 & 4 & 41000 & -2 & 07900 \\
-560 & 29400 & -3 & 76320 & 14 & 11200 & -20 & 16000 & 9 & 70200 \\
1260 & -52920 & 7 & 05600 & -27 & 21600 & 39 & 69000 & -19 & 40400 \\
-1260 & 44100 & -6 & 04800 & 23 & 81400 & -35 & 28000 & 17 & 46360 \\
462 & -13860 & 1 & 94040 & -7 & 76160 & 11 & 64240 & -5 & 82120
\end{array}\right)
$$

Certain interesting properties of these inverses have already been pointed out before. The following features of the $A_{n}{ }^{-1}$ are also noteworthy:

a. It is seen that

$$
\operatorname{sgn} \alpha_{i j}^{(n)}=\left\{\begin{array}{l}
(-1)^{i+j-1} \text { for } i \geq 1, j \geq 1, \text { for } n \text { even } \\
(-1)^{i+j-1} \text { for } i \geq 1, j \geq 2, \text { for } n \text { odd } \\
(-1)^{i+j} \text { for } i \geq 1, j=1, \text { for } n \text { odd. }
\end{array}\right.
$$

b. $\left\|A_{n}{ }^{-1}\right\|=\alpha_{k+1, k+1}^{(n)}$ or $\alpha_{k, k+1}^{(n)}$ with $k+1<n$ for $n>3$.

Now

$$
\left\|A_{n}{ }^{-1}\right\| \geq\left|\alpha_{n n}^{(n)}\right| \sim C_{1} 2^{4 n} .
$$

Inspection of the $\left\|A_{n}{ }^{-1}\right\|$ suggests that

$$
\left\|A_{n}{ }^{-1}\right\| \sim C_{2} 2^{5 n} \text { with } \quad C_{2} \approx 4 \cdot 10^{-3} .
$$

With these estimates it is seen that the measures $M\left(A_{n}\right)$ are approximately

$$
M\left(A_{n}\right) \sim C_{2} n 2^{5 n},
$$

so that the order of ill-conditioning is about that of Hilbert's matrix.

The exact values of the $M\left(A_{n}\right)$ are shown in Table $1 \mathrm{~b}$.

It has been stated [6] that "average" matrices have $M$-condition numbers of the order $n^{1 / 2} \log n$, and $P$-condition numbers of the order $n$. In the light of such orders for condition numbers the matrices $A_{n}$ must indeed be called extremely pathological.

There is a large number of techniques in matrix algebra which are specifically restricted to symmetric positive matrices $H$; for the use of such procedures there have been calculated the matrices

$$
H_{n}=A_{n}{ }^{T} A_{n} .
$$

From the reciprocals of $A_{n}$ those of $H_{n}$ are easily calculated as

$$
H_{n}^{-1}=A_{n}^{-1}\left(A_{n}^{-1}\right)^{T} \text {. }
$$

The inverses $H_{n}{ }^{-1}$ thus also consist of integer elements only. It is known (TAUSSKY [8]) that the condition (in several senses) of $A A^{T}$ is worse than that of $A$.

Obviously in $H_{k+1}=\left(h_{i j}^{(k+1)}\right)$,

$$
\begin{array}{rlrl}
h_{i j}^{(k+1)} & =1+[(i+1)(j+1)]^{-1}+[(i+2)(j+2)]^{-1}+\cdots \\
& =h_{i j}^{(k)}+[(i+k)(j+k)]^{-1} & +[(i+k)(j+k)]^{-1}
\end{array}
$$


for all $i, j \leq k, k=1,2, \cdots$, with $h_{i j}^{(1)}=1$. Consequently,

$$
h_{i i}^{(k+1)}=1+\Psi^{\prime}(i)-\sum_{j=k+1}^{\infty}(i+j)^{-2},
$$

where $\Psi(x)=\Gamma^{\prime}(x+1) / \Gamma(x+1)$. Further, for fixed $k$,

(3.5) $\max _{i, j} h_{i j}^{(k)}=h_{11}^{(k)}=1+\Psi^{\prime}(1)-\sum_{j=k}^{\infty}(1+j)^{-2}=\pi^{2} / 6-\sum_{j=k}^{\infty}(i+j)^{-2}$.

A listing of $H_{6}$ is provided in Table 3.

TABLE 3. The matrix $H_{6}$

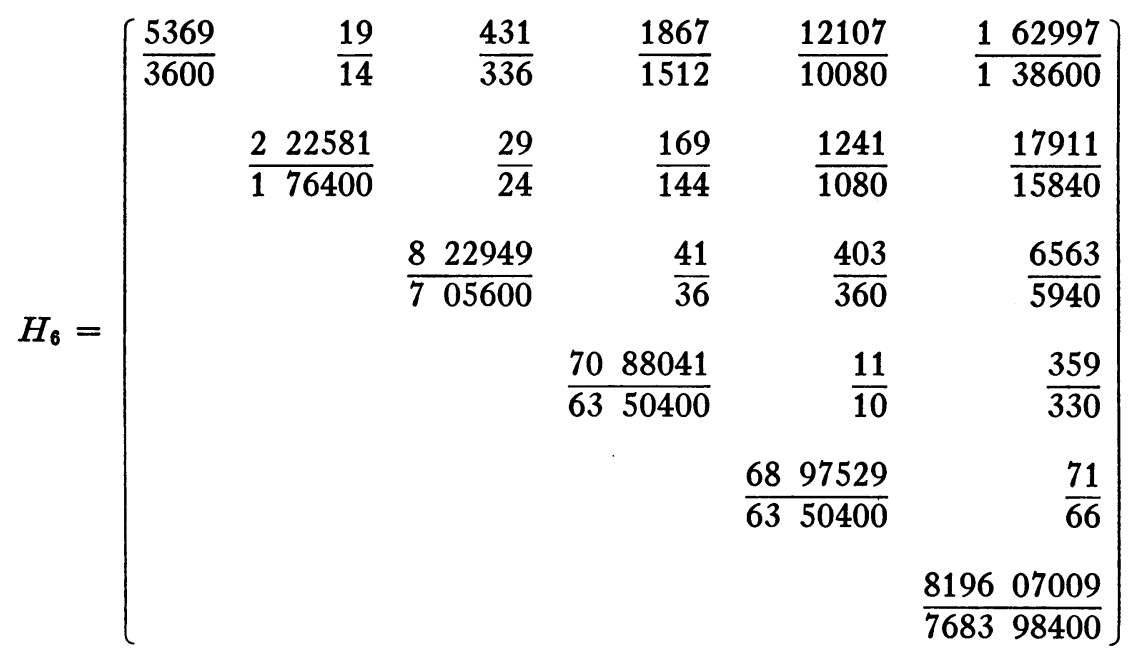

4. Characteristic roots and vectors. Since there is a unique characteristic root $\lambda_{M}{ }^{(n)}$ of largest absolute value the power method [7] may be used to isolate $\lambda_{M}{ }^{(n)}$, while simultaneously obtaining the associated characteristic vector $x_{M}^{(n)}$ :

$$
\begin{gathered}
A_{n} x_{k-1}^{(n)}=\lambda_{k}^{(n)} x_{k}^{(n)}, \quad k=1,2,3, \cdots \\
\lim _{k \rightarrow \infty} \lambda_{k}^{(n)}=\lambda_{M}{ }^{(n)}, \quad \lim _{k \rightarrow \infty} x_{k}^{(n)}=x_{M}^{(n)},
\end{gathered}
$$

TABLE 4. Characteristic roots

\begin{tabular}{rcc}
$n$ & $\lambda_{M}^{(n)}$ & \multicolumn{1}{c}{$\lambda_{m}^{(n)}$} \\
1 & 1.000000 & \\
2 & 1.448403 & -.1150693 \\
3 & 1.707105 & $-.4815399 \cdot 10^{-2}$ \\
4 & 1.886632 & $-.1441324 \cdot 10^{-3}$ \\
5 & 2.022999 & $-.4489833 \cdot 10^{-5}$ \\
6 & 2.132376 & $-.1397499 \cdot 10^{-6}$ \\
7 & 2.223362 & $-.4336577 \cdot 10^{-8}$ \\
8 & 2.301055 & $-.1340623 \cdot 10^{-9}$ \\
9 & 2.368717 & $-.4129309 \cdot 10^{-11}$ \\
10 & 2.428554 & $-.1267649 \cdot 10^{-12}$
\end{tabular}




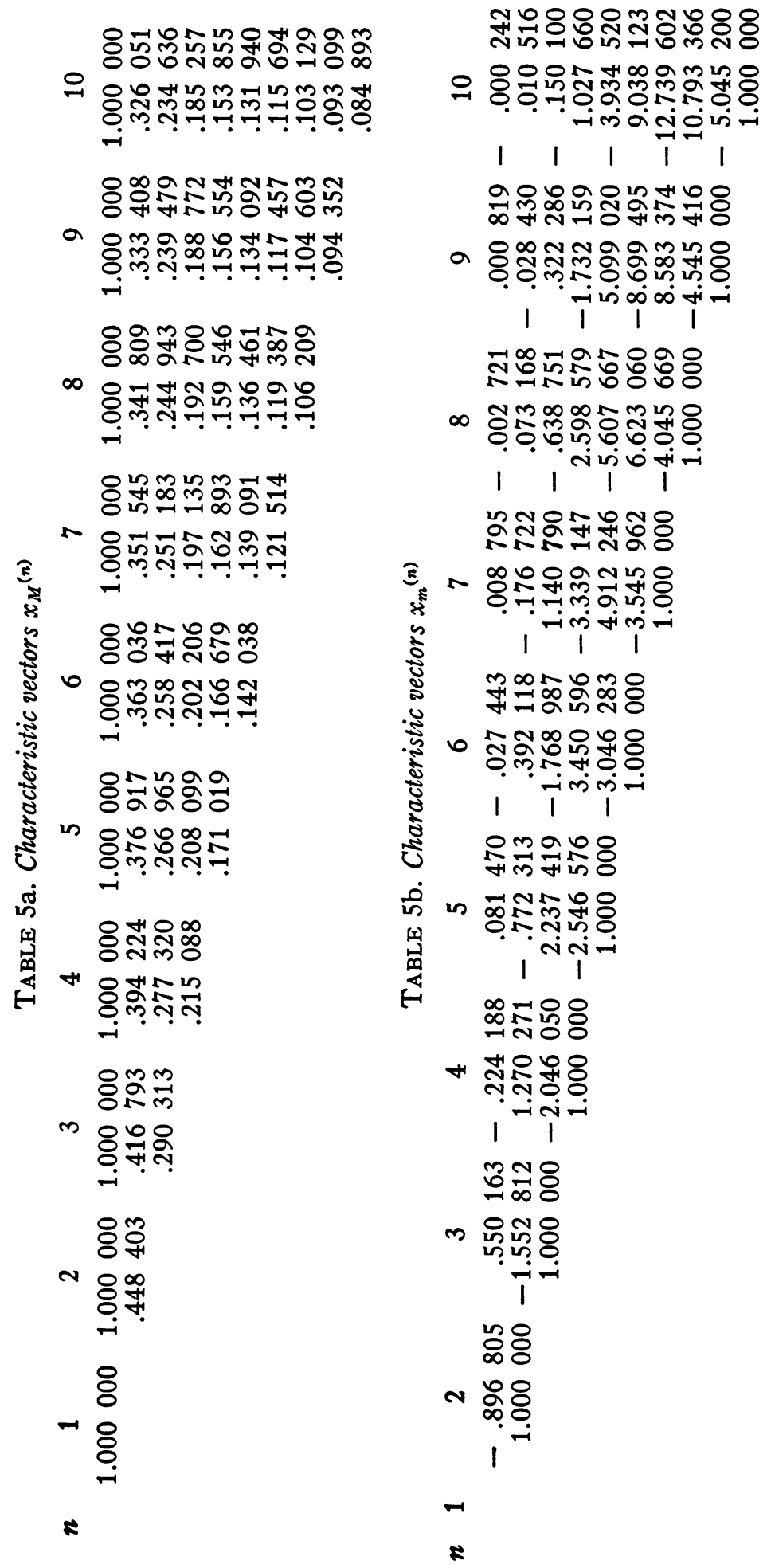


provided the initial arbitrary vector $x_{0}{ }^{(n)}$ is not orthogonal to $x_{M}{ }^{(n)}$. The rate of convergence increases with the ratio between $\lambda_{M}{ }^{(n)}$ and the next characteristic root of $A_{n}$.

The absolutely largest elements of $A_{n}$ occur in the first row; it is therefore advisable to normalize the $x_{M}^{(n)}$ by keeping its first element equal to unity. In carrying out the computation a considerable saving in labor may be further achieved by utilizing the values of $x_{M}{ }^{(n)}$ obtained at the $n$th step to start the next step with a vector $x_{0}^{(n+1)}$ whose first $n$ components were identical with $x_{M}^{(n)}$. Proceeding in this manner there was obtained in seven or less iterations an agreement to six decimal places in both characteristic roots and vectors.

The characteristic roots $\lambda_{M}{ }^{(n)}$ are shown in the middle column of Table 4; as indicated by (2.6) they actually do not grow faster than $\log n$.

The characteristic vectors $x_{M}^{(n)}$ belonging to the $\lambda_{M}{ }^{(n)}$ are listed in Table 5a. Their smallest components $x_{M n}^{(n)}$ seem to decrease as $n^{-1}$. The bounds given in (2.7) thus seem a bit wide.

The characteristic roots $\lambda_{m}{ }^{(n)}=\mu_{M}{ }^{(n)}$ of least absolute value were also calculated, together with their characteristic vectors $x_{m}{ }^{(n)}$, now normalized to have their last component equal to unity. The values of $\lambda_{m}{ }^{(n)}$ are shown in the third column of Table 4 . Here the inequality (2.8), with $\xi=0$, is found to restrict the roots fairly well, the upper bounds - $\min \left(S^{(n)}, T^{(n)}\right)^{-1}$ being, at least for $n \leq 10$, less than 30 per cent too high.

For purposes of comparison the resulting condition numbers $P\left(A_{n}\right)$ were also computed; they are exhibited in the last column of Table $1 \mathrm{~b}$. These suggest the following rough estimate:

$$
P\left(A_{n}\right) \sim C_{3} 2^{5 n} \log n, \quad C_{3} \approx 8 \cdot 10^{-3},
$$

which, with (3.2), gives,

$$
P\left(A_{n}\right) \sim(2 / n) \log n M\left(A_{n}\right) ;
$$

Todd [1] found for Hilbert's matrix $B$

$$
\pi n^{-1} M\left(B_{n}\right)<P\left(B_{n}\right)<\pi M\left(B_{n}\right) .
$$

AVCO Manufacturing Corporation

Stratford, Connecticut

1. JOHN TODD, "The condition of the finite segments of the Hilbert Matrix," in Contributions to the Solution of Systems of Linear Equations and the Determination of Eigenvalues, NBS Applied Math. Series No. 39, 1954, p. 109-116.

2. G. FROBENIUS, "Uber Matrizen aus positiven Elementen," Sitzungsberichte Kgl. Preuss. Akad. Wiss., 1908, p. 471-476; 1909, p. 514-518.

3. W. V. PARKER, "Characteristic roots and the field of values of a matrix," Duke Math. Jn., v. 15,1948 , p. $439-442$.

4. A. OstrowsKI, "Bounds for the greatest latent root of a positive matrix," London Math. Soc., Jn., v. 27, 1952 , p. 253-256.

5. MARK LOTKIN \& RUSSELl REMAGE, "Scaling and error analysis for matrix inversion by partitioning," Ann. Math. Stat., 24, 1953, p. 428-439.

6. JoHn ToDd, "The condition of a certain matrix," Cambridge Phil. Soc., Proc., v. 46, 1949, p. $116-118$.

7. R. A. Frazer, W. J. Duncan, \& A. R. Collar, Elementary Matrices, Cambridge Univ. Press, 1950.

8. Olga TAussky, "Note on the condition of matrices," MTAC, v. 4, 1940, p. 111-112. 